\title{
Immunological Hypoglycemia Associated with Insulin Antibodies Induced by Exogenous Insulin in 11 Chinese Patients with Diabetes
}

\author{
Heng Quan, Huiwen Tan, Qianrui Li, Jianwei Li, and Sheyu Li \\ Department of Endocrinology and Metabolism, West China Hospital of Sichuan University, Chengdu, Sichuan 610041, China
}

Correspondence should be addressed to Jianwei Li; jerryli678@yahoo.com and Sheyu Li; lisheyu@gmail.com

Received 20 January 2015; Revised 26 March 2015; Accepted 30 March 2015

Academic Editor: Mitsuhiko Noda

Copyright (C) 2015 Heng Quan et al. This is an open access article distributed under the Creative Commons Attribution License, which permits unrestricted use, distribution, and reproduction in any medium, provided the original work is properly cited.

\begin{abstract}
Aims. To investigate the characteristics of immunological hypoglycemia associated with insulin antibodies (IAbs) induced by exogenous insulin in Chinese patients with diabetes. Methods. The clinical data of patients with immunological hypoglycemia due to IAbs were retrospectively analyzed by screening patients with diabetes discharged from West China Hospital from 2007 to 2013. Results. A total of 11 patients (eight men and three women) were identified. Insulin-C-peptide separation was found in all patients via insulin and C-peptide release test. Previous insulin use was ceased after admission and was switched to oral hypoglycemic agents (OHAs) (8/11), lifestyle modification only (2/11), or regular human insulin (1/11). Hypoglycemia was ameliorated after a median of 20 days (interquartile range [IQR], 11-40), while IAbs turned negative after a median of 17 months (IQR, 4-19), and serum immunoreactive insulin (IRI) levels dropped substantially after a median of 22 months (IQR, 9-32) in these cases. Conclusions. In insulin-treated patients with unexpected and refractory hypoglycemia even after insulin therapy was gradually reduced or even withdrawn, IAbs induced by exogenous insulin should be considered, and insulin withdrawal might be promptly needed. The course of immunological hypoglycemia was benign and self-limited.
\end{abstract}

\section{Introduction}

Hypoglycemia is one of the most important complications of diabetes. It is commonly observed in diabetic patients on insulin therapy and also occurs occasionally after the cessation of insulin. Immunological hypoglycemia associated with insulin antibodies (IAbs) induced by exogenous insulin was first reported by Harwood in 1960 [1]. It is a relatively rare but important subtype of unexpected hypoglycemia in insulin-treated patients with diabetes even after they have stopped insulin administration. It has been poorly described, usually ignored, and difficult to manage. Little experience has been summarized before.

In the present study, we tended to retrospectively analyze the clinical characteristics of a cohort of patients with hypoglycemia associated with IAbs induced by exogenous insulin in a tertiary referral center, to help clinicians better understand the manifestation and management of such condition.

\section{Subjects and Methods}

2.1. Study Design and Patients. We retrospectively reviewed 6,824 patients with diabetes discharged from the Department of Endocrinology and Metabolism in West China Hospital of Sichuan University from January 2007 to December 2013.

Patients were eligible for inclusion in the study if they had hypoglycemia as one of the discharge diagnoses, received insulin therapy prior to admission with positive IAb, and had available results from blood glucose monitoring, standard meal tolerance test (MTT), and insulin and C-peptide release test during hospitalization. Patients were excluded if they had self-reported ever use of methimazole, tiopronin, glutathione, or other thiol-containing agents or had other established causes of hypoglycemia, such as excessive use of oral hypoglycemic agents (OHAs) or insulin.

Additionally, we selected patients hospitalized during the same period with negative IAb and no record of unexpected 
hypoglycemia episodes, matched to each case patient by insulin type, age (within a 3-year range), and gender in a 1:1 ratio, as controls to evaluate the effects of the agents' crossreaction in the insulin test.

Our study was carried out in accordance with the Declaration of Helsinki and Good Clinical Practice guidelines.

2.2. Data Collection. The demographic profiles, clinical characteristics, and laboratory data of all subjects were collected. All laboratory tests were conducted in the Department of Laboratory Medicine in West China Hospital of Sichuan University. Standard MTT and insulin and C-peptide release test began at 08:00 a.m. after a 10-hour overnight fast. Blood samples were drawn for glucose, insulin, and C-peptide determination before and 2 hours after a loading meal containing $75 \mathrm{~g}$ carbohydrates without insulin usage in the morning. Plasma glucose was measured by hexokinase method (Roche, Germany). Levels of serum immunoreactive insulin (IRI) and C-peptide reactivity (CPR) were determined by electrochemiluminescence immunoassay (ECLIA) method (Roche, Germany). Serum alanine transaminase (ALT) and aspartate transaminase (AST) were measured by the method without pyridoxal phosphate activation suggested by the International Federation of Clinical Chemistry (IFCC) (Roche, Germany). Serum creatinine was measured by the Jaffe method (Roche, Germany). Estimated glomerular filtration rate (eGFR) was calculated by the Chronic Kidney Disease Epidemiology Collaboration (CKD-EPI) equation. Insulin antibody (IAb), islet cell antibody (ICA), and glutamic acid decarboxylase antibody (GAD-Ab) were evaluated with enzyme-linked immunosorbent assay (ELISA) method (Biomerica, CA, USA). Each sample was run in duplicate, and optical density (OD) was measured in an ELISA reader at $405 \mathrm{~nm}$. An index was calculated based on the average of the results of each sample for IAb and ICA: Index $=(\mathrm{OD}$ of test sample)/(OD of quality control reference). The "positive IAb" or "positive ICA" was defined as an index > 1.05 (test sample $\mathrm{A}_{405 \mathrm{~nm}}$ /quality control reference $\left.\mathrm{A}_{405 \mathrm{~nm}}>1.05\right)$. The "negative IAb" or "negative ICA" was defined as an index $<0.95$ (test sample $\mathrm{A}_{405 \mathrm{~nm}} /$ quality control reference $\left.\mathrm{A}_{405 \mathrm{~nm}}<0.95\right)$. The "positive GAD-Ab" and "negative GAD-Ab" were defined as an average value of GAD-Ab $>1.05 \mathrm{U} / \mathrm{mL}$ and $<1.00 \mathrm{U} / \mathrm{mL}$, respectively. If the value stayed critical (0.95-1.05 for IAb and ICA, $1.00-1.05 \mathrm{U} / \mathrm{mL}$ for GAD-Ab), we repeated the test or drew new parallel sample and tested again. IAb, ICA, and $\mathrm{GAD}-\mathrm{Ab}$ of the patients were recorded as positive or negative without exact value. Blood glucose was monitored by ACCUCHEK Active nine times per day (preprandially, $2 \mathrm{~h}$ postprandially, 10:00 p.m., 0:00 a.m., and 3:00 a.m.) for all patients since admission, and if hypoglycemic symptoms occurred at any other time, blood glucose would also be monitored immediately. Type of hypoglycemia, namely, preprandial or postprandial (defined as 2 hours from meal), and at night (defined as between 10:00 p.m. and 06:00 a.m.), was classified as the time range when more than half of hypoglycemic episodes occurred. Unexpected hypoglycemia was identified as refractory hypoglycemic episodes occurring 3 days after the cessation of insulin.
2.3. Statistical Analysis. Continuous variables including age, body mass index (BMI), course of diabetes, duration of insulin treatment, ALT, AST, creatinine, eGFR, glycated hemoglobin ( $\mathrm{HbAlc}$ ), and the lowest blood glucose were presented as medians and interquartile ranges (IQR) due to an inadequate sample size and uncertain distribution. Categorical variables including episodes of hypoglycemia and insulin usage were presented as frequencies. Analyses were performed with Origin software, version 8.0 (OriginLab) and Statistical Package for Social Sciences 20.0 (SPSS, Chicago, IL). Paired chi-square test was used in comparing dichotomous variables between groups, when Wilcoxon signed rank test was used in comparing continuous data. All statistical tests were two-tailed and $P<0.05$ was considered statistically significant.

\section{Results}

A total of 11 patients with unexpected hypoglycemia (eight men and three women) were eventually identified with a median age of 70 years (IQR, 61-77). The median course of diabetes was 48 months (IQR, 12-120). The median serum ALT, AST, and eGFR were $26 \mathrm{IU} / \mathrm{L}$ (IQR, 1538), $24 \mathrm{IU} / \mathrm{L}$ (IQR, 15-35), and $88.07 \mathrm{~mL} / \mathrm{min} / 1.73 \mathrm{~m}^{2}$ (IQR, 74.48-99.64), respectively (Table 1). Compared with patients without unexpected hypoglycemia, patients with unexpected hypoglycemia had a smaller BMI $\left(21.00 \mathrm{~kg} / \mathrm{m}^{2}\right.$ [IQR, 19.9021.64] versus $25.15 \mathrm{~kg} / \mathrm{m}^{2}$ [IQR, 20.06-29.07], $P=0.036$ ), a trend of shorter duration of insulin treatment without statistical significance (5 months [IQR, 4-12] versus 36 months [IQR, 24-60], $P=0.050$ ), lower insulin dosage (15 IU [IQR, 14-20] versus $26 \mathrm{IU}$ [IQR, 24-30], $P=0.016)$, lower fasting PG (5.93 mmol/L [IQR, 4.52-6.30] versus $8.89 \mathrm{mmol} / \mathrm{L}$ [IQR, 7.19-12.17], $P=0.041$ ), lower HbAlc (6.4\% [IQR, 5.97.3] versus $8.3 \%$ [IQR, 6.7-11.8], $46 \mathrm{mmol} / \mathrm{mol}$ [IQR, 41-51] versus $67 \mathrm{mmol} / \mathrm{mol}$ [IQR, 50-105], $P=0.041)$, higher fasting and after MTT $2 \mathrm{~h}$ IRI (hi pmol/L [IQR, 2437.20-hi] versus $69.12 \mathrm{pmol} / \mathrm{L}$ [IQR, 29.82-93.42], $P=0.003$; hi pmol/L [IQR, hi-hi] versus $187.62 \mathrm{pmol} / \mathrm{L}$ [IQR, 113.88-339.36], $P=$ 0.003; hi was defined as insulin $>6000.00 \mathrm{pmol} / \mathrm{L})$ and CPR (1.810 nmol/L [IQR, 1.010-3.130] versus $0.768 \mathrm{nmol} / \mathrm{L}$ [IQR, 0.462-0.905], $P=0.010 ; 3.350 \mathrm{nmol} / \mathrm{L}$ [IQR, 3.100-4.220] versus $1.470 \mathrm{nmol} / \mathrm{L}$ [IQR, 1.350-1.840], $P=0.003)$, and higher frequency of hypoglycemia (3 episodes/week [IQR, 15] versus 1 episode/week [IQR, 0-1], $P=0.007$ ) (Table 3).

All the patients received insulin therapy for blood glucose control prior to admission, and the insulin strategies of each patient were shown in Table 1 . The median dose of insulin in patients with unexpected hypoglycemia was 15 IU (IQR, 1416) per day. Seven patients received premixed insulin, while the other four received rapid acting insulin. As shown in Table 1, after a median time of 5 months (IQR, 4-12) from the initiation of insulin therapy, unexpected hypoglycemia occurred recurrently in these patients even after discontinued insulin therapy. Six patients (cases 4-7, 10, and 11) had more frequent hypoglycemia during night than during daytime, and the frequent day-time hypoglycemia of the other five patients was usually preprandial. The median lowest 


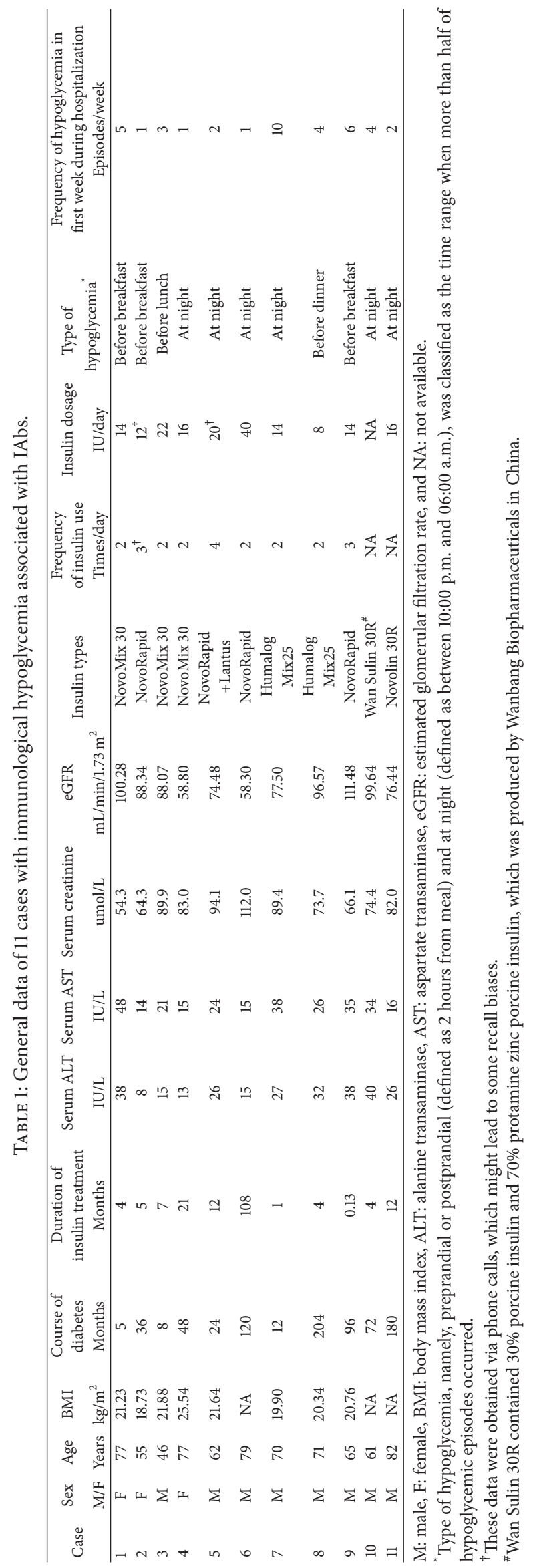


blood glucose monitored was $2.0 \mathrm{mmol} / \mathrm{L}$ (IQR, 1.3-2.7). Four patients (cases 3, 6, 7, and 11) experienced disorders of consciousness such as confusion or coma. And one patient (case 7) receiving Humalog Mix25 also experienced insulin allergy presented as rashes, redness, and itches at the injection site. For all patients with unexpected hypoglycemia, insulin overdose was the initially suspected cause of hypoglycemia. Thus the dose of insulin was gradually reduced in all these patients or even discontinued in six patients (cases 2, 3, 5, 7,8 , and 11) by patients themselves or by physicians prior to admission. However, spontaneous hypoglycemia occurred recurrently even after discontinuation of insulin, and thus they were admitted to the hospital for further examination and management.

Their laboratory tests after admission indicated positive IAb in all patients with unexpected hypoglycemia, positive ICA in case 6 , and positive GAD-Ab in case 10 . The fasting and $2 \mathrm{~h}$ IRI concentrations after MTT significantly increased in these patients, while the C-peptide levels were relatively low, which was described as insulin-C-peptide separation (Table 2). However, insulin-C-peptide separation was not observed in patients without unexpected hypoglycemia and their IRI concentrations were much lower (Table 3), which indicated a low cross-reaction of the test reagent with exogenous insulin. Therefore, the increased serum IRI in patients with unexpected hypoglycemia could be considered endogenous and the hypoglycemic attacks might be due to IAb-insulin dissociation.

To relieve hypoglycemia, the previously used insulin was reduced and then withdrawn in all patients who developed unexpected hypoglycemia after admission, with replacement of other blood glucose control strategies. Cases 3, 4, 5, 8, and 9 received $\alpha$-glucosidase inhibitor 3 times per day instead, case 1 received glinide twice per day (before breakfast and lunch), case 11 received $\alpha$-glucosidase inhibitor in combination with biguanide, and case 6 received triple drugs, namely, glinide, $\alpha$-glucosidase inhibitor, and dipeptidyl peptidase-IV (DPPIV) inhibitor. Case 10 switched to regular recombinant human insulin from premixed insulin. Cases 2 and 7 only received diet control and advice to exercise after previous insulin withdrawal. Moreover, case 7, who experienced both hypoglycemia and allergy, received glucocorticoid therapy. Methylprednisolone was infused intravenously $40 \mathrm{mg} / \mathrm{d}$ for 3 days, followed by oral prednisone at $30 \mathrm{mg} / \mathrm{d}$. Prednisone was reduced gradually and discontinued in 16 days, when the allergic skin reaction was alleviated as well.

After cessation of insulin, hypoglycemia was still observed recurrently during hospitalization (Table 1), although it improved generally in these patients in the present study. The median time when hypoglycemic episodes vanished during hospitalization after previous insulin withdrawal was 20 days (IQR, 11-40).

Seven patients with unexpected hypoglycemia were followed up after discharge. Six of them (cases 2-5, 8, and 9) got complete remission of hypoglycemia, while case 1 had a significant reduction of the frequency of hypoglycemia (only two episodes before breakfast during the 9-month follow-up). IAbs turned negative in four cases (cases 3, 4, 8, and 9) after a median of 17 months (IQR, 4-19). HbAlc was $44.5 \mathrm{mmol} / \mathrm{mol}$
(IQR, 42-50) or 6.25\% (IQR, 6.0-6.7) in six cases (cases 2-5, 8 , and 9 ) after a median of 23.5 months (IQR, 15-31). Standard MTTs and insulin and C-peptide release tests were conducted during follow-up in six cases (cases 1-3, 5, 8, and 9), and serum IRI levels substantially dropped after a median of 22 months (IQR, 9-32) (Table 2).

\section{Discussion}

Hypoglycemia is a common problem in diabetic patients with antidiabetic therapies, especially in those receiving insulin treatment. It is highly associated with mortality and cardiovascular events as illustrated by a number of studies [2-5]. Most episodes of hypoglycemia occurred due to insulin overdose or irregular food intake and could be easily prevented by insulin dose adjustment or lifestyle modification. However, hypoglycemia due to other causes could also present refractory after modification of insulin dose and food intake. In the current study, patients presented with unexpected hypoglycemia even after discontinuation of insulin and with obvious insulin-C-peptide separation in insulin and C-peptide release test. Thus, insulin autoimmune hypoglycemia due to IAbs should be considered, and etiological treatment should be given to diminish the episodes [6].

As established already, IAbs could be induced by methimazole and other thiol-containing agents $[7,8]$, as well as exogenous insulin injection $[9,10]$. Methimazole-induced $\mathrm{IAb}$ is highly associated with unexpected hypoglycemia and insulin resistance and could be difficult to treat, requiring glucocorticoid and other immunosuppressive treatments in most cases [11, 12]. IAb induced by exogenous insulin is common in insulin-treated patients, especially in those years when human insulin was not derived and used in clinical practice [13-15]. However, it is not always associated with hypoglycemia or insulin resistance, and IAb-associated hypoglycemia is usually reported as single cases in the literature [16-18].

To our knowledge, the present report is the largest case series of IAb-associated immunological hypoglycemia in China, indicating that IAb is of clinical significance and should be considered in patients with persistent and unexplained hypoglycemia even after insulin withdrawal, especially in old male with long-term use of rapid insulin analogues or neutral protamine Hagedorn (NPH).

The present study (Table 1) showed that insulin analogues and NPH could be associated with IAbs production in insulin-treated patients with diabetes. Insulin analogues, which differ from human insulin, have been proved to cause IAbs response $[15,19,20]$. NPH, a heterologous protein, could be antigenic itself and is also an adjuvant to increase the antigenicity of exogenous insulin $[21,22]$.

The accuracy of insulin measurement affected the results. In the present study, insulin concentration was measured by the ECLIA method with a monoclonal antibody, which reported a cross-reactivity of $<0.02 \%$ for insulin aspart and insulin lispro [23, 24], 19.2\% for porcine insulin, and 0.05\% for proinsulin (PI) (provided by the instruction of kit). Additionally, blood samples in all patients were drawn for IRI determination without insulin usage in the morning to 


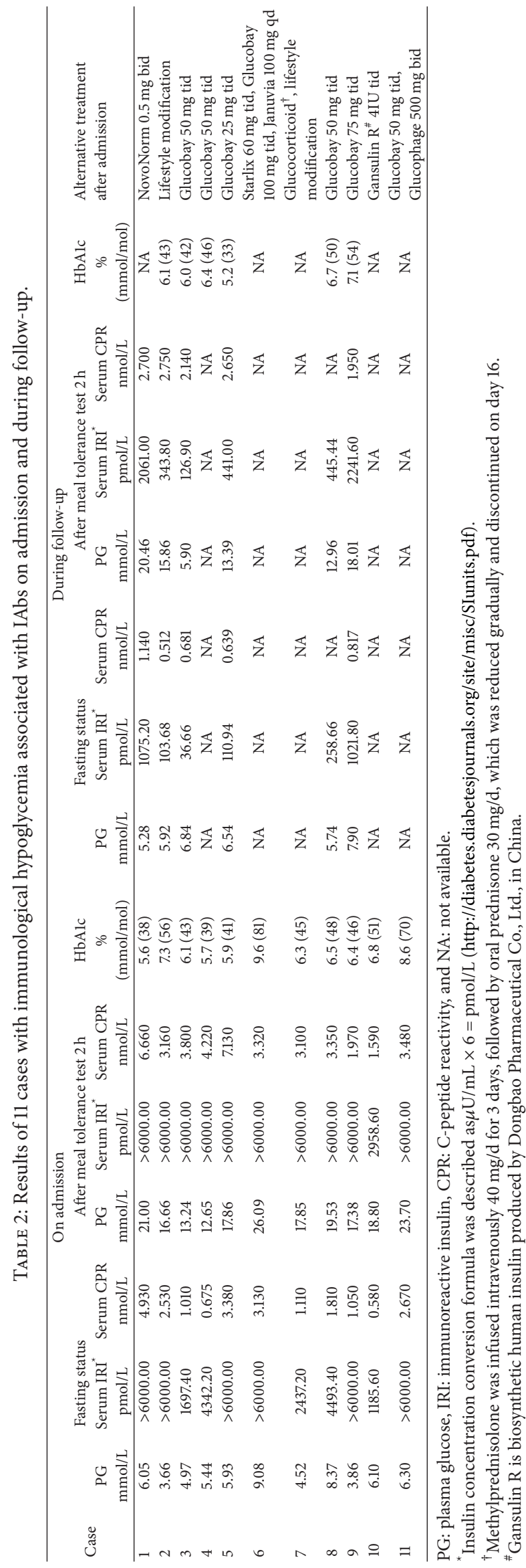


TABLE 3: Characteristics of the patients with unexpected hypoglycemia and patients without unexpected hypoglycemia.

\begin{tabular}{|c|c|c|c|}
\hline Variable & $\begin{array}{l}\text { Patients with unexpected } \\
\text { hypoglycemia }(n=11)\end{array}$ & $\begin{array}{l}\text { Patients without unexpected } \\
\text { hypoglycemia }(n=11)\end{array}$ & $P$ value \\
\hline Male (\%) & $8(72.73)$ & $8(72.73)$ & 1.000 \\
\hline Age, median (IQR), years & $70(61-77)$ & $70(60-77)$ & 0.608 \\
\hline BMI, median (IQR), $\mathrm{kg} / \mathrm{m}^{2}$ & $21.00(19.90-21.64)$ & $25.15(20.06-29.07)$ & 0.036 \\
\hline Course of diabetes, median (IQR), months & $48(12-120)$ & $120(84-156)$ & 0.139 \\
\hline Duration of insulin treatment, median (IQR), months & $5(4-12)$ & $36(24-60)$ & 0.050 \\
\hline Serum ALT, median (IQR), IU/L & $26(15-38)$ & $23(20-34)$ & 0.722 \\
\hline Serum AST, median (IQR), IU/L & $24(15-35)$ & $25(23-30)$ & 0.683 \\
\hline Serum creatinine, median (IQR), umol/L & $82.0(66.1-89.9)$ & $88.0(74.0-106.0)$ & 0.328 \\
\hline eGFR, median (IQR), $\mathrm{mL} / \mathrm{min} / 1.73 \mathrm{~m}^{2}$ & $88.07(74.48-99.64)$ & $72.49(51.05-93.96)$ & 0.374 \\
\hline Insulin dosage, median (IQR), IU/day & $15(14-20)$ & $26(24-30)$ & 0.016 \\
\hline \multicolumn{4}{|l|}{ Fasting status on admission } \\
\hline PG, median (IQR), mmol/L & $5.93(4.52-6.30)$ & $8.89(7.19-12.17)$ & 0.041 \\
\hline Serum IRI ${ }^{*}$ median (IQR), pmol/L & hi $(2437.20-h i)^{\dagger}$ & $69.12(29.82-93.42)$ & 0.003 \\
\hline Serum CPR, median (IQR), nmol/L & $1.810(1.010-3.130)$ & $0.768(0.462-0.905)$ & 0.010 \\
\hline \multicolumn{4}{|l|}{ After meal tolerance test $2 \mathrm{~h}$ on admission } \\
\hline PG, median (IQR), mmol/L & $17.86(16.66-21.00)$ & $19.34(16.20-21.14)$ & 0.657 \\
\hline Serum IRI ${ }^{*}$ median (IQR), pmol/L & hi $(\text { hi-hi) })^{\dagger}$ & $187.62(113.88-339.36)$ & 0.003 \\
\hline Serum CPR, median (IQR), nmol/L & $3.350(3.100-4.220)$ & $1.470(1.350-1.840)$ & 0.003 \\
\hline \multicolumn{4}{|l|}{ HbAlc } \\
\hline median (IQR), \% & $6.4(5.9-7.3)$ & $8.3(6.7-11.8)$ & \multirow{2}{*}{0.041} \\
\hline median (IQR), $\mathrm{mmol} / \mathrm{mol}$ & $46(41-51)$ & $67(50-105)$ & \\
\hline $\begin{array}{l}\text { Frequency of hypoglycemia in first week during } \\
\text { hospitalization, median (IQR), episodes/week }\end{array}$ & $3(1-5)$ & $1(0-1)$ & 0.007 \\
\hline
\end{tabular}

reduce cross-reaction. Furthermore, patients without unexpected hypoglycemia were recorded with a larger insulin dosage and a lower IRI concentration than patients with unexpected hypoglycemia, suggesting that the extremely high levels of IRI in patients with unexpected hypoglycemia could not be explained by exogenous insulin. Consequently, the measured IRI in our study could be considered the true insulin level [25], which included IAb-binding insulin and free insulin [23]. Although the C-peptide test kit with a monoclonal antibody had a cross-reactivity of $32.5 \%$ for PI, PI concentrations in fasting status were 100 times less than C-peptide concentration (provided by the instruction of kit), and the measured CPR was the true C-peptide level as well [26].

The genetic basis of IAb-associated immunological hypoglycemia was not fully understood. Human leukocyte antigen (HLA) was reported to play a role and individuals with HLADR4 genotype were susceptible to develop IAb-associated immunological hypoglycemia $[27,28]$, resembling that of a patient with insulin autoimmune syndrome (IAS) $[8,29]$, type 1 diabetes mellitus $[30,31]$, or rheumatoid arthritis $[32,33]$. However, HLA typing was not performed in this retrospective study.
To be clarified, two types of IAbs have been revealed by previous studies: high affinity with low capacity and low affinity with high capacity $[34,35]$. And the IAbs in patients with immunological hypoglycemia induced by exogenous insulin has been found to be the latter type [28]. The circulating IAbs may act as an insulin binding reservoir, impacting the amount of biologically active insulin and leading to higher postprandial blood glucose levels after a subcutaneous insulin injection, but when a large portion of insulin dissociates from the IAbs, this process is independent of the plasma glucose level, leading to the onset of unexpected hypoglycemia [27, 36].

Our results also showed that IAbs could turn negative in most patients after insulin withdrawal given sufficient followup duration, which was consistent with a previous study [37] demonstrating that IAbs might disappear one year after insulin withdrawal. This indicated that IAb was not a lifelong antibody, and immunological hypoglycemia was benign and self-limited. All our patients withdrew previous insulin to minimize antigen stimulation, which was of importance in the treatment of immunological hypoglycemia, and most of them received OHAs as replacement, if the residual $\beta$ cell function could supply the essential insulin demands 
of the patients. However, transient hypoglycemia should be alerted due to the release of previously combined insulin from the IAb-insulin complex when the autoantibody was erased. Generally, the hypoglycemia attacks relieved in parallel with the decrease in the titer of the IAb and serum insulin level after insulin therapy was discontinued.

As ICA and GAD-Ab were detected in cases 6 and 10, respectively, latent autoimmune diabetes in adults (LADA) could be suspected when taking into consideration their history of diabetes and their laboratory tests. Patients with LADA were insulin-free for at least six months after diagnosis because of tolerable postprandial C-peptide concentration and they could be managed by OHAs in this period of time [38]. Case 6 used glinide (starlix) to replace insulin administration and his glycemic control was improved upon discharge. However, he was lost to follow-up and thus later condition was unknown, although LADA patients using controversial agents, such as glinide, needed long-term observation. The present case suggested that clinicians should be cautious with the choice of OHAs for patients with LADA and with rigorous follow-up.

On the other hand, some patients would have to change insulin type if they had a low postprandial C-peptide concentration such as case 10 who was diagnosed with diabetes for six years. Of note, the changing of insulin type was proved to be effective. Recombinant human insulin, an ideal alternative, has least antigenicity but could still lead to IAbs production, either because of the fact that the insulin injected in a foreign site was immunogenic or because of the occurrence of subcutaneous polymerization [39-41]. Therefore, OHAs are better for non-insulin-dependent diabetic patients than insulin, because of its effects on minimizing the possibility of IAbs induced by exogenous insulin.

Glucocorticoid may be helpful in extremely serious cases or in patients hypersensitive to insulin. Glucocorticoid could work dramatically and was supposed to be withdrawn within weeks. Immunosuppressive agents or plasmapheresis could be another option for severe cases according to previous reports $[28,42]$.

IAbs could be considered a complication of the treatment with exogenous insulin. As a result, IAb-associated hypoglycemia should be considered when initiating insulin therapy. Most of the patients in our case series ceased insulin therapy and glycemic control remained satisfying, indicating that nonessential prescription of insulin was common in Chinese patients with diabetes. Proper use of exogenous insulin, especially insulin analogs, should be reconsidered by both diabetologists and general practitioners.

The present study has several limitations. Firstly, this is a single-centered retrospective observational and descriptive study with a limited number of patients and a limited duration of follow-up. Patients with positive IAb but without episodes of unexpected hypoglycemia were not recruited in this study. Secondly, information from telephone follow-up was occasionally used in the analysis of two cases (cases 2 and 5), which might lead to some recall biases. Thirdly, insulinoma-associated protein 2 (IA-2) antibody, the exact titer of IAb, and IAb affinity and capacity were not measured, and thus it was difficult to quantify the change of titers of IAbs in these patients. Therefore, further well-designed larger prospective cohort studies are still to be suggested with predefined endpoints of laboratory and clinical parameters, including IA-2 antibody, the exact titer of IAb, affinity and capacity of IAb, and HLA typing.

\section{Conclusions}

In summary, hypoglycemia in insulin-treated patients with diabetes might result from various causes, among which immunological causes associated with IAbs shall not be neglected, although it is a rare etiology and insulin overdose should always be addressed before considering other causes. If unexpectedly recurrent hypoglycemia occurred, especially after insulin had been discontinued for several days, immunological hypoglycemia associated with IAbs due to exogenous insulin should be considered. Lab tests for insulin concentration, C-peptide concentration, and IAb titer could help make diagnosis. Metformin, $\alpha$-glucosidase inhibitor, and DPP-IV inhibitors appeared to be alterative blood glucose control options for minimizing the risk of hypoglycemia.

\section{Disclosure}

This study has never been published previously.

\section{Conflict of Interests}

No potential conflict of interests relevant to this paper was reported.

\section{Authors' Contribution}

Heng Quan and Huiwen Tan contributed equally to this work. Jianwei $\mathrm{Li}$ conceived and designed the study and oversaw the analyses. Heng Quan and Huiwen Tan performed data extraction and analyses, participated in data analysis planning and interpretation of study findings, and wrote the paper for publication. Sheyu Li participated in data analysis planning and interpretation of study findings, and revised the paper for submission for publication. Qianrui Li participated in revising the paper for submission for publication. Jianwei $\mathrm{Li}$ is the guarantor of this work and, as such, has full access to all the data in the study and takes responsibility for the integrity of the data and the accuracy of the data analysis.

\section{Acknowledgments}

This study was funded by the National Natural Science Foundation of China (NSFC Grant no. 30600293 and Grant no. 81400811$)$.

\section{References}

[1] R. Harwood, "Insulin-binding antibodies and 'spontaneous' hypoglycemia," The New England Journal of Medicine, vol. 262, no. 19, pp. 978-979, 1960. 
[2] A. Goto, O. A. Arah, M. Goto, Y. Terauchi, and M. Noda, "Severe hypoglycaemia and cardiovascular disease: systematic review and meta-analysis with bias analysis," British Medical Journal, vol. 347, no. 7919, Article ID f4533, 11 pages, 2013.

[3] H. C. Gerstein, M. E. Miller, R. P. Byington et al., "Effects of intensive glucose lowering in type 2 diabetes," The New England Journal of Medicine, vol. 358, no. 24, pp. 2545-2559, 2008.

[4] W. Duckworth, C. Abraira, and T. Moritz, "Glucose control and vascular complications in veterans with type 2 diabetes," The New England Journal of Medicine, vol. 360, no. 2, pp. 129-139, 2009.

[5] S. Finfer, D. R. Chittock, S. Y. Su et al., "Intensive versus conventional glucose control in critically ill patients," The New England Journal of Medicine, vol. 360, no. 13, pp. 1283-1297, 2009.

[6] C. Jaeger, M. Eckhard, M. D. Brendel, and R. G. Bretzel, "Diagnostic algorithm and management of immune-mediated complications associated with subcutaneous insulin therapy," Experimental and Clinical Endocrinology and Diabetes, vol. 112, no. 8, pp. 416-421, 2004.

[7] N. Furukawa, N. Miyamura, K. Nishida, H. Motoshima, K. Taketa, and E. Araki, "Possible relevance of alpha lipoic acid contained in a health supplement in a case of insulin autoimmune syndrome," Diabetes Research and Clinical Practice, vol. 75, no. 3, pp. 366-367, 2007.

[8] Y. Uchigata, Y. Hirata, and Y. Iwamoto, "Drug-induced insulin autoimmune syndrome," Diabetes Research and Clinical Practice, vol. 83, no. 1, pp. e19-e20, 2009.

[9] T. W. van Haeften, B. A. Krom, and J. E. Gerich, "Prolonged fasting hypoglycemia due to insulin antibodies in patient with non-insulin-dependent diabetes mellitus: Effect of insulin withdrawal on insulin-antibody-binding kinetics," Diabetes Care, vol. 10, no. 2, pp. 160-163, 1987.

[10] S. G. Albert and D. A. Popp, "Hypoglycemia due to serumcomplexed insulin in a patient with diabetes mellitus," Diabetes Care, vol. 7, no. 3, pp. 285-290, 1984.

[11] Y. Uchigata, Y. Eguchi, S. Takayama-Hasumi, and Y. Omori, "Insulin autoimmune syndrome (Hirata disease): clinical features and epidemiology in Japan," Diabetes Research and Clinical Practice, vol. 22, no. 2-3, pp. 89-94, 1994.

[12] M. J. Gomez Cruz, M. Jabbar, N. Saini et al., "Severe hypoglycemia secondary to methimazole-induced insulin autoimmune syndrome in a 16 year old African-American male," Pediatric Diabetes, vol. 13, no. 8, pp. 652-655, 2012.

[13] S. E. Fineberg, T. T. Kawabata, D. Finco-Kent, R. J. Fountaine, G. L. Finch, and A. S. Krasner, "Immunological responses to exogenous insulin," Endocrine Reviews, vol. 28, no. 6, pp. 625652, 2007.

[14] R. Wredling, P. E. Lins, and U. Adamson, "Prevalence of antiinsulin antibodies and its relation to severe hypoglycaemia in insulin-treated diabetic patients," Scandinavian Journal of Clinical and Laboratory Investigation, vol. 50, no. 5, pp. 551-557, 1990.

[15] T. W. Van Haeften, "Clinical significance of insulin antibodies in insulin-treated diabetic patients," Diabetes Care, vol. 12, no. 9, pp. 641-648, 1989.

[16] A. Matsuyoshi, S. Shimoda, K. Tsuruzoe et al., "A case of slowly progressive type 1 diabetes with unstable glycemic control caused by unusual insulin antibody and successfully treated with steroid therapy," Diabetes Research and Clinical Practice, vol. 72, no. 3, pp. 238-243, 2006.
[17] T.-Y. Zhao, F. Li, and Z.-Y. Xiong, "Frequent reoccurrence of hypoglycemia in a type 2 diabetic patient with insulin antibodies," Molecular Diagnosis and Therapy, vol. 14, no. 4, pp. 237-241, 2010.

[18] S. Salardi, E. Cacciari, L. Steri et al., "An 8-year follow-up of anti-insulin antibodies in diabetic children: relation to insulin autoantibodies, HLA type, $\beta$-cell function, clinical course and type of insulin therapy," Acta Paediatrica, vol. 84, no. 6, pp. 639645, 1995.

[19] P. Raskin, R. A. Guthrie, L. Leiter, A. Riis, and L. Jovanovic, "Use of insulin aspart, a fast-acting insulin analog, as the mealtime insulin in the management of patients with type 1 diabetes," Diabetes Care, vol. 23, no. 5, pp. 583-588, 2000.

[20] S. E. Fineberg, J. Huang, R. Brunelle, K. S. Gulliya, and J. H. Anderson Jr., "Effect of long-term exposure to insulin lispro on the induction of antibody response in patients with type 1 or type 2 diabetes," Diabetes Care, vol. 26, no. 1, pp. 89-96, 2003.

[21] R. Mylvaganam, J. M. Stowers, J. M. Steel, J. Wallace, J. C. MacHendry, and A. D. Wright, "Insulin immunogenicity in pregnancy: maternal and fetal studies," Diabetologia, vol. 24, no. 1, pp. 19-25, 1983.

[22] J. M. Weiler, M. A. Gellhaus, J. G. Carter et al., "A prospective study of the risk of an immediate adverse reaction to protamine sulfate during cardiopulmonary bypass surgery," Journal of Allergy and Clinical Immunology, vol. 85, no. 4, pp. 713-719, 1990.

[23] R. Sapin, V. Le Galudec, F. Gasser, M. Pinget, and D. Grucker, "Elecsys insulin assay: free insulin determination and the absence of cross-reactivity with insulin lispro," Clinical Chemistry, vol. 47, no. 3, pp. 602-605, 2001.

[24] W. E. Owen and W. L. Roberts, "Cross-reactivity of three recombinant insulin analogs with five commercial insulin immunoassays," Clinical Chemistry, vol. 50, no. 1, pp. 257-259, 2004.

[25] C. Saddig, P. E. Goretzki, and A. A. R. Starke, "Differentiation of insulin secretion patterns in insulinoma," World Journal of Surgery, vol. 32, no. 5, pp. 918-929, 2008.

[26] J. P. Cassidy, S. D. Luzio, M. T. Marino, and R. A. Baughman, "Quantification of human serum insulin concentrations in clinical pharmacokinetic or bioequivalence studies: what defines the 'best method'?" Clinical Chemistry and Laboratory Medicine, vol. 50, no. 4, pp. 663-666, 2012.

[27] K. Suzuki, S. Hirayama, and S. Ito, "A case of a non-insulin dependent diabetic patient with regular spontaneous hypoglycemic attacks, which were due to insulin-binding antibodies induced by human insulin therapy," Tohoku Journal of Experimental Medicine, vol. 182, no. 2, pp. 163-173, 1997.

[28] T. Ishizuka, S. Ogawa, T. Mori et al., "Characteristics of the antibodies of two patients who developed daytime hyperglycemia and morning hypoglycemia because of insulin antibodies," Diabetes Research and Clinical Practice, vol. 84, no. 2, pp. e21e23, 2009.

[29] Y. Uchigata, "The novel agent, alpha lipoic acid, can cause the development of insulin autoimmune syndrome," Internal Medicine, vol. 46, no. 17, pp. 1321-1322, 2007.

[30] M. P. Morran, A. Vonberg, A. Khadra, and M. Pietropaolo, "Immunogenetics of type 1 diabetes mellitus," Molecular Aspects of Medicine, 2015.

[31] K. A. McLaughlin, K. Gulati, C. C. Richardson et al., "HLADR4-associated $\mathrm{T}$ and $\mathrm{B}$ cell responses to specific determinants on the IA-2 autoantigen in type 1 diabetes," The Journal of Immunology, vol. 193, no. 9, pp. 4448-4456, 2014. 
[32] V. Taneja, "Cytokines pre-determined by genetic factors are involved in pathogenesis of rheumatoid arthritis," Cytokine, 2014.

[33] J. U. Scher and S. B. Abramson, "Periodontal disease, Porphyromonas gingivalis, and rheumatoid arthritis: what triggers autoimmunity and clinical disease?" Arthritis Research and Therapy, vol. 15, no. 5, article 122, 2013.

[34] M. R. Kim, L. R. Sheeler, N. Mansharamani, M. T. Haug, C. Faiman, and M. K. Gupta, "Insulin antibodies and hypoglycemia in diabetic patients: can a quantitative analysis of antibody binding predict the risk of hypoglycemia?" Endocrine, vol. 6, no. 3, pp. 285-291, 1997.

[35] B. M. Brooks-Worrell, D. Nielson, and J. P. Palmer, "Insulin autoantibodies and insulin antibodies have similar binding characteristics," Proceedings of the Association of American Physicians, vol. 111, no. 1, pp. 92-96, 1999.

[36] S. Oak, T.-H. T. Phan, L. K. Gilliam, I. B. Hirsch, and C. S. Hampe, "Animal insulin therapy induces a biased insulin antibody response that persists for years after introduction of human insulin," Acta Diabetologica, vol. 47, no. 2, pp. 131-135, 2010.

[37] C. Ionescu-Tîrgovite, I. Mincu, L. Simionescu et al., "Disappearance rate of insulin antibodies after discontinuing insulin treatment in 42 type 2 (non-insulin-dependent) diabetic patients," Diabetologia, vol. 27, no. 6, pp. 592-595, 1984.

[38] S. Fourlanos, F. Dotta, C. J. Greenbaum et al., "Latent autoimmune diabetes in adults (LADA) should be less latent," Diabetologia, vol. 48, no. 11, pp. 2206-2212, 2005.

[39] S. E. Fineberg, J. A. Galloway, N. S. Fineberg, M. J. Rathbun, and S. Hufferd, "Immunogenicity of recombinant DNA human insulin," Diabetologia, vol. 25, no. 6, pp. 465-469, 1983.

[40] H. G. Velcovsky and K. F. Federlin, "Insulin-specific IgG and IgE antibody response in type I diabetic subjects exclusively treated with human insulin (recombinant DNA)," Diabetes Care, vol. 5, no. 2, pp. 126-128, 1982.

[41] M. Iavicoli, U. Di Mario, G. A. Coronel, A. M. Dawud, P. Arduini, and M. Leonardi, "Semisynthetic human insulin: biologic and immunologic activity in newly treated diabetic subjects during a six-month follow-up," Diabetes Care, vol. 7, no. 2, pp. 128-131, 1984.

[42] R. Koyama, K. Nakanishi, M. Kato, S. Yamashita, H. Kuwahara, and H. Katori, "Hypoglycemia and hyperglycemia due to insulin antibodies against therapeutic human insulin: treatment with double filtration plasmapheresis and prednisolone," The American Journal of the Medical Sciences, vol. 329, no. 5, pp. 259264, 2005. 


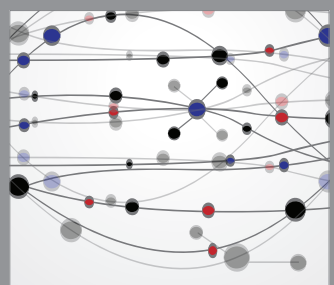

The Scientific World Journal
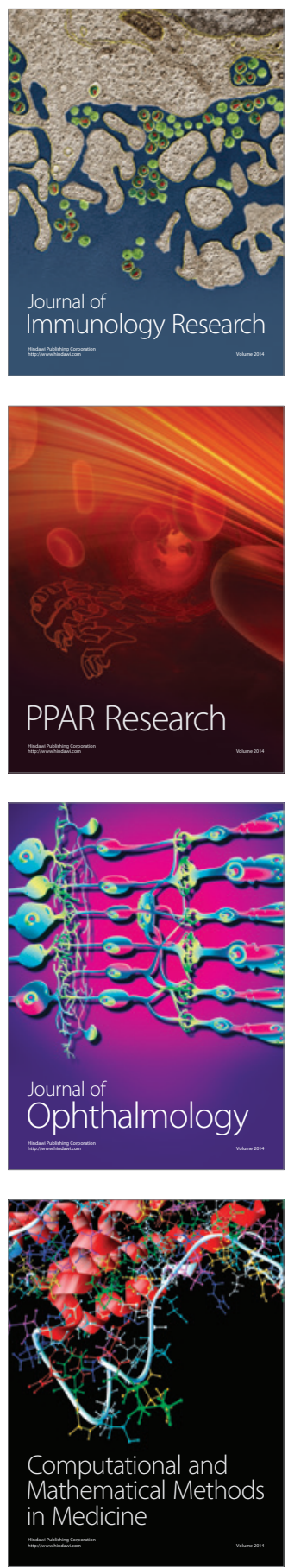

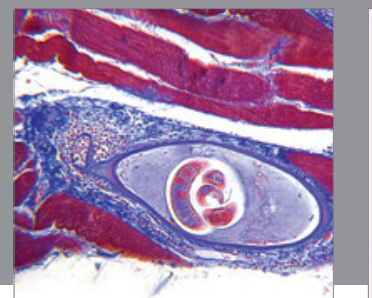

Gastroenterology

Research and Practice
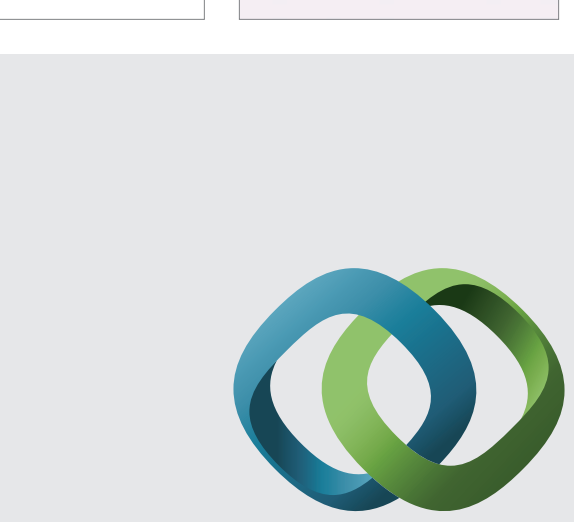

\section{Hindawi}

Submit your manuscripts at

http://www.hindawi.com
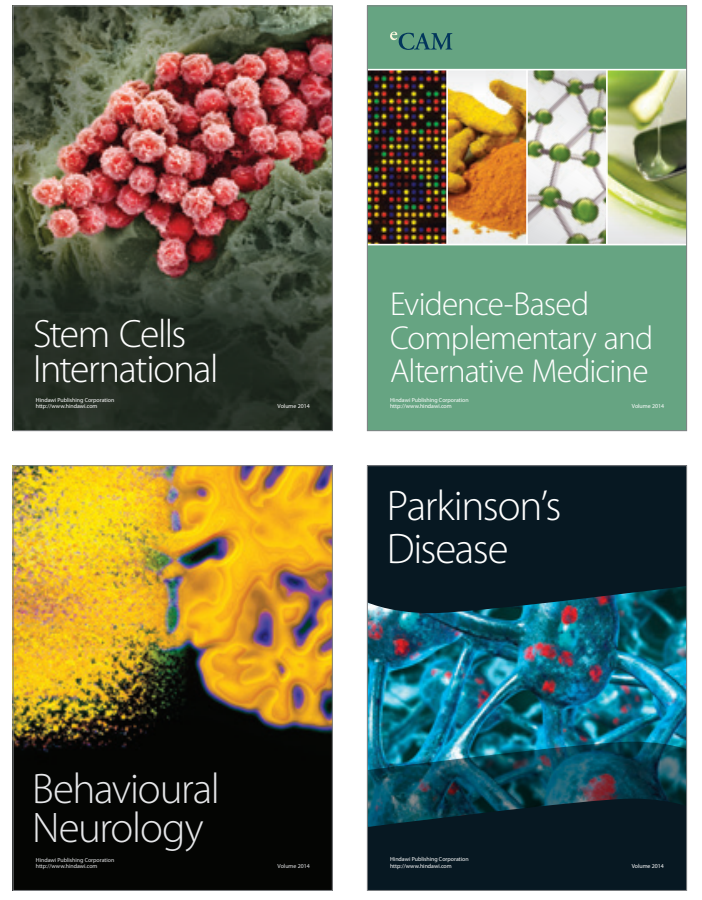
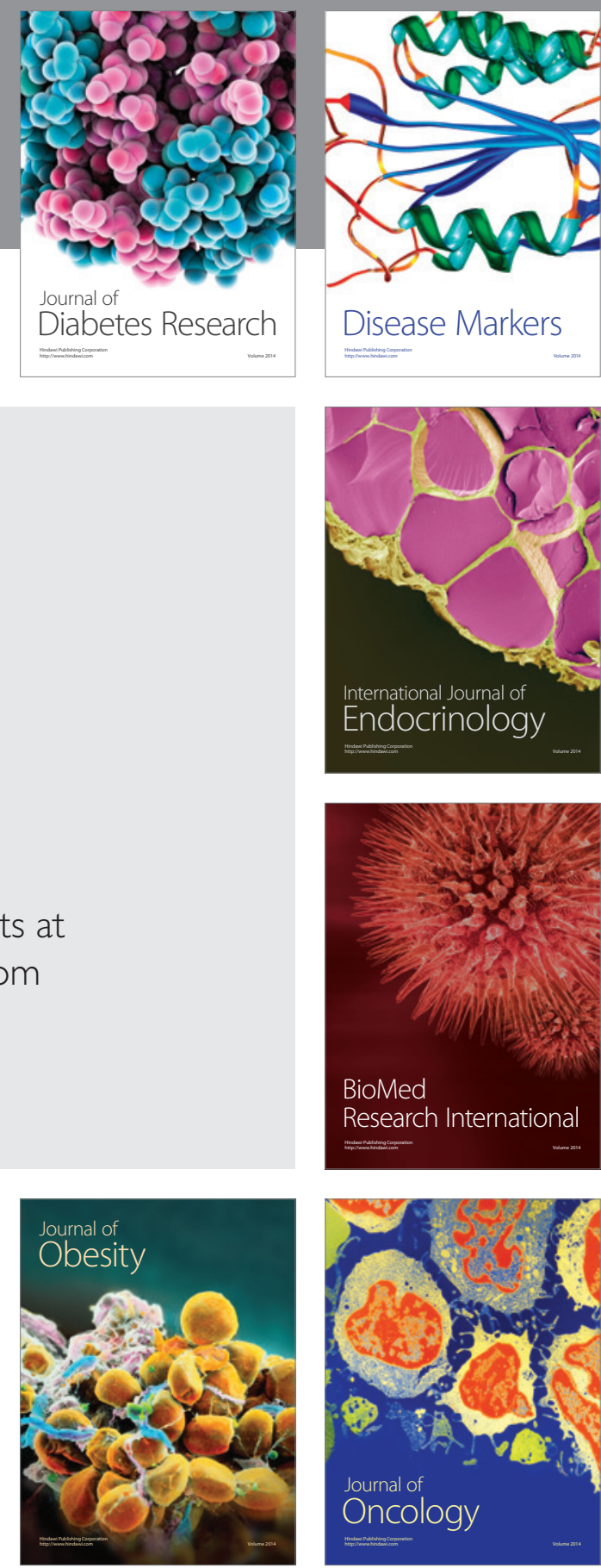

Disease Markers
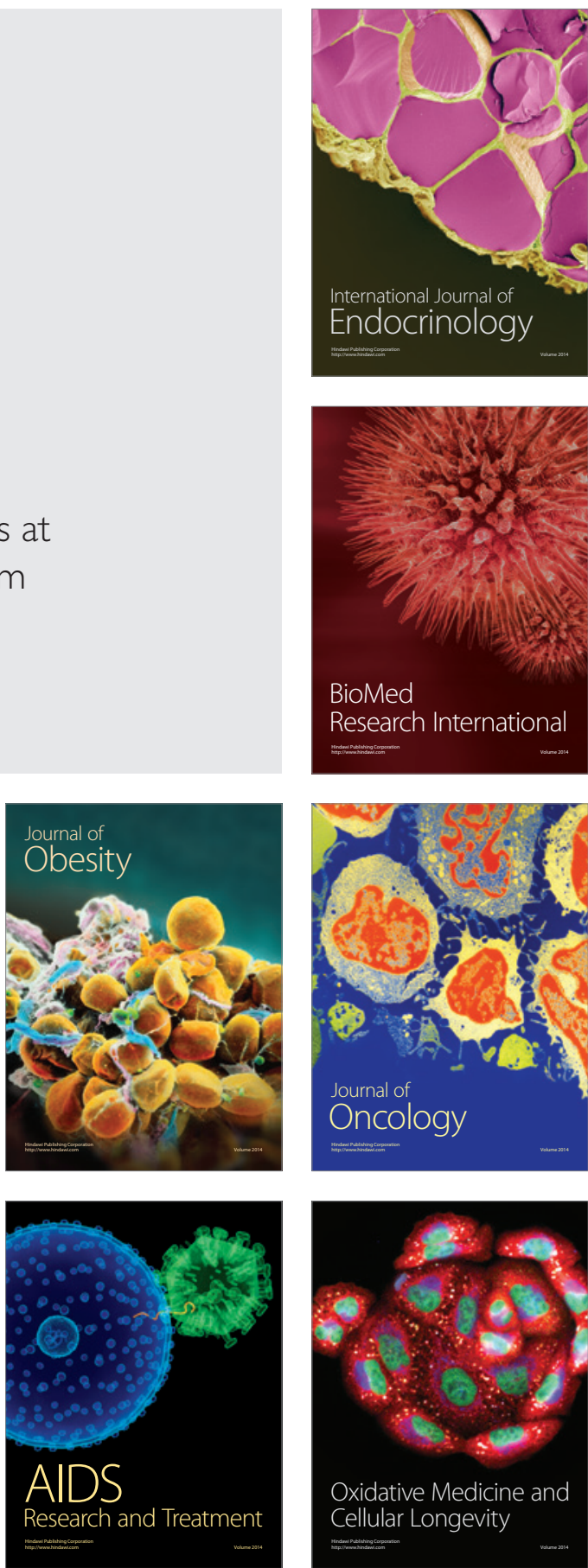